hep-th/9901143

TUW-99-01

\title{
String Corrections to the Hawking-Page Phase Transition
}

\author{
Karl Landsteiner \\ Institut für theoretische Physik \\ Technische Universität Wien, TU-Wien \\ Wiedner Hauptstraße 8-10 \\ A-1040 Wien, Austria \\ landstei@brane.itp.tuwien.ac.at
}

\begin{abstract}
We compute the $O\left(\alpha^{\prime 3}\right)$ corrections to the $A d S_{5}$ Black hole metric in type IIB string theory. Contrary to previous work in this direction we keep the Black Hole radius finite. Thus the topology of the boundary is $S^{3} \times S^{1}$. We find the corrections to the free energy and the critical temperature of the phase transition.
\end{abstract}

January, 1999

Research supported by the FWF under project P13125-PHY. 
The study of large $N$ gauge theories received in the last year new input from Maldacena's conjecture that type IIB superstring theory on asymptotically AdS - backgrounds is dual to the strong coupling limit of large $N$ gauge theories [1]. Originally the conjecture states that type IIB string theory on $A d S_{5} \times S^{5}$ is dual to $\mathcal{N}=4$ supersymmetric gauge theory with gauge group $S U(N)$ in the limit of large $N$ and strong 't Hooft coupling $\lambda=g_{Y M}^{2} N$. The string loop expansion corresponds to the $1 / N$ expansion of the gauge theory and the sigma-model $\alpha$ ' expansion to the expansion in the 't Hooft coupling. Denoting the scale of the $A d S_{5} \times S^{5}$ geometry by $\ell$, the AdS/CFT dictionary says $\frac{\ell^{4}}{\alpha^{\prime 2}}=\lambda$ and $g_{\text {string }}=4 \pi g_{Y M}^{2}$. The gauge theory is thought to live on the conformal boundary of the $A d S$ space. Further specification of the conjecture has been achieved in [2], in particular it was shown how to calculate correlation functions in the boundary conformal field theory. The conjecture was very soon generalized by Witten to gauge theories at finite temperature [3]. He observed that replacing the AdS space by the AdS Schwarzschild metric should correspond to have the gauge theory at finite temperature on $S^{3}$. Taking the scaling limit of a large black hole one can make the radius of the three sphere infinite. The metric obtained in this way coincides with the near horizon limit of the near extremal threebrane solution [4]. The relation between the near extremal threebrane solution and gauge theories at finite temperature has been studied already earlier by Klebanov et al. [5]. In particular they found that the Bekenstein-Hawking entropy of the threebrane solution reproduces the entropy of the $\mathcal{N}=4$ gauge theory up to a relative factor of $\frac{3}{4}$. It is now understood that this difference stems from the fact, that the calculation in the gauge theory is done at vanishing coupling, whereas the string calculation is relevant to the regime of infinite coupling (see the recent review by Klebanov [6]). In [7] the authors calculated the $O\left(\alpha^{\prime 3}\right)$ correction to the entropy stemming from the $R^{4}$ term in the effective action of type IIB stringst. This calculation has actually a somewhat involved history. It was first done using only the five dimensional limit of the AdS Schwarzschild metric which is asymptotic to $S^{1} \times R^{3}$ in two different ways. First the authors plugged in the uncorrected expression for the metric in the $R^{4}$ term. In a second step they also calculated the corrections to the metric. Quite surprisingly the results for the free energy turned out to agree. It was then pointed out in [9] that one actually has to consider the whole ten dimensional metric including the $S^{5}$ factor. However, the thermodynamic quantities turned out to be unchanged in comparison to the purely five dimensional calculation. For the latter fact an explanation has been offered in an appendix to [7]. Using

1 Corrections stemming from these terms have first been conisered in context of the Ads/CFT correspondence in [8]. 
the AdS/CFT dictionary this correction translates into the next to leading order term in a strong coupling expansion in $\frac{1}{\lambda}$ at infinite $N$. On general grounds one expects for the entropy $S=\frac{2 \pi^{2}}{3} N^{2} V_{3} T^{3} f(\lambda)$. The strong coupling expansion corresponding to the string theory regime gives then $f(\lambda)=\frac{3}{4}+\frac{45}{32} \zeta(3)(2 \lambda)^{-3 / 2}+\cdots$. A recent field theory two loop calculation gives the expansion around $\lambda=0$ as $f(\lambda)=1-\frac{3}{2 \pi^{2}} \lambda+\cdots$ [10].

Let us turn now to the AdS Schwarzschild black hole which asymptotically approaches $S^{3} \times S^{1}$. The ten dimensional metric used as IIB background is given by

$$
d s^{2}=\frac{r^{2}}{\ell^{2}} e^{-\frac{10}{3} C(r)}\left(e^{2 A(r)+8 B(r)} d \tau^{2}+e^{2 B(r)} d r^{2}+d \Omega_{3}^{2}\right)+e^{2 C(r)} d \Omega_{5}^{2} .
$$

We have chosen here the analog of the parameterization as in the appendix of [7], which turns out to be very convenient for calculating the string corrections. The $O\left(\alpha^{\prime 0}\right)$ expression for the functions $A(r), B(r)$ and $C(r)$ are

$$
\begin{aligned}
& A(r)=-2 \log \left(\frac{r}{\ell}\right)+\frac{5}{2} \log \left(\frac{r^{2}}{\ell^{2}}+\frac{r^{4}}{\ell^{4}}-\frac{r_{0}^{2}}{\ell^{4}}\right) \\
& B(r)=-\frac{1}{2} \log \left(\frac{r^{2}}{\ell^{2}}+\frac{r^{4}}{\ell^{4}}-\frac{r_{0}^{2}}{\ell^{4}}\right) ; \\
& C(r)=0 .
\end{aligned}
$$

Here $r_{0}$ is a parameter determining the mass of the black hole. The thermodynamical properties of the four dimensional AdS Schwarzschild black hole have been investigated some years ago by Hawking and Page [1]. For the five dimensional case at hand things are completely parallel. We summarize the relevant facts. The metric does not have a conical singularity if $\tau$ is compactified on a circle of radius $\beta=\frac{2 \pi r_{+} \ell^{2}}{2 r_{+}^{2}+\ell^{2}}$. This defines the Hawking temperature $T=\frac{1}{\beta}$ and $r_{+}$is the largest root of $r^{2} \ell^{2}+r^{4}+r_{0}^{4}=0$. For a given temperature there are two solutions

$$
r_{+}=\frac{\pi T \ell^{2}}{2}\left(1 \pm \sqrt{1-\frac{2 \ell^{2}}{\pi^{2} T^{2}}}\right)
$$

the smaller black hole is however unstable against decay into the larger one. The black hole solution exists only down to a minimal temperature $T_{\min }=\frac{\sqrt{2}}{\ell \pi}$. The action is calculated by subtracting the value with $r_{0}=0$ which corresponds to empty Anti-de-Sitter space. It changes sign at $r_{+}=\ell\left(T_{c}=\frac{3}{2 \ell \pi}\right)$ thereby signalling a phase transition. For $r_{+} \leq \ell$ the black hole is unstable against tunneling into pure AdS space. Witten argued that this behavior corresponds to a large $\mathrm{N}$ high/low temperature phase transition in the gauge theory on the boundary [3]. At low temperature the theory is described by AdS-space. The 
free energy is of order one indicating a confining phase. At high temperature the AdS black hole geometry is thermodynamically favored. The free energy is of order $N^{2}$ indicating a deconfining phase of the gauge theory. This high/low temperature phase transition occurs only when the gauge theory lives on $S^{3}$. Actually, because of the conformal symmetry of the $\mathcal{N}=4$ gauge theory the behavior depends only on the dimensionless quantity $\frac{\beta}{\ell}$, measuring the ratio of the radius of the circle to the radius of the three sphere. We are interested in calculating the $O\left(\alpha^{\prime 3}\right)$ corrections to the relevant thermodynamical quantities. This question has been addressed before in [12]. There the authors did not consider corrections to the geometry but just plugged in the uncorrected metric into the $R^{4}$ term. This is a somewhat oversimplified procedure since it ignores the corrections to the geometry. It seems therefore worth to reconsider the problem taking into account also the $O\left(\alpha^{\prime 3}\right)$ corrections to the metric. For notational convenience we will set the AdS scale $\ell=1$, it can be reintroduced easily by dimensional analysis.

We use the action

$$
S=\frac{1}{16 \pi G_{10}} \int d^{10} x \sqrt{-g_{10}}\left(R-\frac{1}{2}(\partial \Phi)^{2}-\frac{1}{4.5 !}\left(F_{5}\right)^{2}+\gamma e^{-\frac{3}{2} \Phi} W\right) .
$$

with

$$
\gamma=\frac{1}{8} \zeta(3) \alpha^{\prime 3}
$$

$W$ denotes the $R^{4}$ term and can be expressed as a particular contraction of four Weyltensors

$$
W=C_{a b c d} C^{e b c f} C^{a}{ }_{g h e} C_{f}{ }^{g h d}+\frac{1}{2} C_{a d b c} C^{e f b c} C^{a}{ }_{g h e} C_{f}{ }^{g h d} .
$$

For the metric at $O(0)$ we have $W=\frac{180 r_{0}^{8}}{r^{16}}$. The action for IIB five form field should be understood in the sense that one first varies the action and then implements the selfduality condition at the level of the equation of motion. Such a procedure is convenient for purposes of dimensional reduction. In particular using the ansatz (1) we obtain the one dimensional action

$$
\begin{aligned}
S= & \frac{\operatorname{Vol}\left(S^{3}\right) \operatorname{Vol}\left(S^{5}\right) \beta}{16 \pi G_{10}} \int d r\left[r^{5} e^{A+5 B-\frac{16}{3} C}\left(20-8 e^{-8 C}\right)+\frac{10}{3}\left(r^{3} C^{\prime} e^{A+3 B}\right)^{\prime}+\right. \\
& e^{A+3 B}\left(6 r^{3} e^{2 B}-4 r-8 r^{2} A^{\prime}-2 r^{3}\left(A^{\prime}\right)^{2}-24 r^{2} B^{\prime}-14 r^{3} A^{\prime} B^{\prime}-24 r^{3}\left(B^{\prime}\right)^{2}-\right. \\
& \left.\left.\frac{40}{3}\left(C^{\prime}\right)^{2}-2 r^{3} A^{\prime \prime}-8 r^{3} B^{\prime \prime}\right)+\gamma \text { W-contribution }\right] .
\end{aligned}
$$

As already in the $R^{3}$ case the $\mathrm{W}$-contribution is to long to be displayed here (approx. 1.5 MB Mathematica output). Let us concentrate for a moment on the scale factor for the $S^{5}$. 
Since it will be of order $\gamma$ we find that its contribution to the action at this order is given by the total derivative term solely.

$$
S_{C}=\left.e^{A+3 B} r^{3} C^{\prime}\right|_{r_{+}} ^{\infty}
$$

This expression vanishes at the horizon $r_{+}$. At infinity it vanishes also provided $C(r)$ goes to zero at least as fast as $\frac{1}{r^{5}}$. We set now $A(r)=A_{0}(r)+\gamma A_{1}(r)$, etc. The equations of motion following from the action are

$$
\begin{aligned}
{\left[\left(r^{4}+r^{2}-r_{0}^{2}\right) B_{1}\right]^{\prime}+\frac{10 r_{0}^{6}}{r^{13}}\left(171 r_{0}^{2}-160 r^{2}-144 r^{4}\right) } & =0 \\
\left(r^{4}+r^{2}-r_{0}^{2}\right) A_{1}^{\prime}-10\left(2 r^{3}+r\right) B_{1}+\frac{10 r_{0}^{6}}{r^{13}}\left(576 r^{4}+565 r^{2}-711 r_{0}^{2}\right) & =0 \\
{\left[\left(r^{5}+r^{3}-r r_{0}^{2}\right) C_{1}^{\prime}\right]^{\prime}-32 r^{3} C_{1}+\frac{135 r_{0}^{8}}{2 r^{13}} } & =0 \\
{\left[\left(r^{4}+r^{2}-r_{0}^{2}\right) \Phi_{1}^{\prime}\right]^{\prime}-\frac{270 r_{0}^{8}}{r^{13}} } & =0 .
\end{aligned}
$$

Despite much effort we did not find a closed solution for $C_{1}$. However, as we just saw, this field does not contribute to the action if it vanishes fast enough at infinity. For very large $r$ the differential equation for $C_{1}$ becomes

$$
\left(r^{5} C_{1}^{\prime}\right)^{\prime}-32 r^{3} C_{1}=0
$$

From this we find $C_{1}(r) \approx \frac{c_{1}}{r^{8}}+c_{2} r^{4}$. As boundary condition we demand that the metric should still be asymptotically AdS and thus $c_{2}=0$. This shows that $C_{1}(r)$ vanishes indeed fast enough and does not contribute to the action. The solutions for the other equations are

$$
\begin{aligned}
A_{1}(r)= & \left(r^{4}+r^{2}-r_{0}^{2}\right)^{-1}\left(\frac{1360 r^{2} r_{0}^{6}+1560 r^{4} r_{0}^{6}-1185 r_{0}^{8}}{2 r^{12}}-\frac{25 r_{0}^{6}\left(15 r_{0}^{2}-8 r_{+}^{2}\right)}{2 r_{+}^{12}}\right) \\
B_{1}(r)= & \left(r^{4}+r^{2}-r_{0}^{2}\right)^{-1}\left(\frac{285 r_{0}^{8}-360 r^{4} r_{0}^{6}-320 r^{2} r_{0}^{6}}{2 r^{12}}+\frac{5 r_{0}^{6}\left(15 r_{0}^{2}-8 r_{+}^{2}\right)}{2 r_{+}^{12}}\right) ; \\
\Phi_{1}(r)= & \frac{45}{4 r_{0}^{4}\left(r_{+}^{2}+1\right)}\left(3 r_{0}^{4}+4 r_{0}^{2}+1\right) \log \left(\frac{2\left(r^{2}+r_{+}^{2}+1\right)}{r^{2}}\right)-\frac{3}{16 r_{0}^{4} r^{12}}\left[\left(180 r_{0}^{4}+240 r_{0}^{2}+60\right) r^{10}+\right. \\
& \left.\left(30 r_{0}^{6}+90 r_{0}^{4}+30 r_{0}^{2}\right) r^{8}+\left(40 r_{0}^{6}+20 r_{0}^{4}\right) r^{6}+15 r_{0}^{6}\left(r_{0}^{2}+1\right) r^{4}+12 r_{0}^{8} r^{2}+10 r_{0}^{10}\right] .
\end{aligned}
$$

Demanding that the corrected metric does not have a conical singularity at the horizon $r_{+}$ we find the period $\beta$ and thus the temperature $T=\frac{1}{\beta}$ to be

$$
T=\frac{2 r_{+}^{2}+1}{2 \pi r_{+}}\left(1+\gamma \frac{10\left(r_{+}^{3}+1\right)^{3}\left(3 r_{+}^{2}-1\right)}{r_{+}^{6}\left(2 r_{+}^{2}+1\right)}\right) .
$$


The action at $O(\gamma)$ is

$$
S_{R}=\left.\frac{\beta \operatorname{Vol}\left(S^{3}\right) \operatorname{Vol}\left(S^{5}\right)}{16 \pi G_{10}}\left(-2 r^{4}-\gamma 30 \frac{r_{0}^{6}\left(4 r^{2}+r_{0}^{2}\right)}{r^{12}}\right)\right|_{r_{+}} ^{r_{\max }} .
$$

We regularize this expression in the usual way by subtracting the action for the metric with $R=0$ and the same asymptotic geometry. This condition demands that the period of the empty AdS space $\beta_{0}$ for large radii is related to the period of the AdS black hole $\beta$ through

$$
\beta_{0} \approx \beta\left(1-\frac{r_{0}^{2}}{2 r^{4}}-\gamma \frac{5\left(r_{+}^{2}+1\right)^{3}\left(7+15 r_{+}^{2}\right)}{2 r_{+}^{4}} \frac{1}{r^{4}}+O\left(\frac{1}{r^{5}}\right)\right) .
$$

We end up with the following expression for the free energy $F=\frac{1}{\beta} \lim _{r_{\max } \rightarrow \infty}\left(S_{R}-S_{R=0}\right)$

$$
F=\frac{\operatorname{Vol}\left(S^{3}\right) \operatorname{Vol}\left(S^{5}\right)}{16 \pi G_{10}}\left[r_{+}^{4}-r_{+}^{2}+\gamma \frac{\left(r_{+}^{2}+1\right)^{3}\left(75 r_{+}^{2}-5\right)}{r_{+}^{4}}\right]
$$

The correction to the critical black hole radius that follows from this expression is

$$
r_{+}^{c}=1-280 \gamma
$$

and the corrected critical temperature is

$$
T_{c}=\frac{3}{2 \pi}-\gamma \frac{60}{\pi} .
$$

We finally want to express the free energy as function of the temperature. To do this we need to invert (12). We write $r_{+}=f_{0}(T)+\gamma f_{1}(T)$ where $f_{0}(T)$ is given by the larger value in (3) and $f_{1}(T)$ turns out as

$$
f_{1}(T)=-\frac{20\left(1+T^{2} \pi^{2}\left(1+\sqrt{1-\frac{2}{T^{2} \pi^{2}}}\right)\right)^{3}\left(-5+3 \pi^{2} T^{2}\left(1+\sqrt{1-\frac{2}{T^{2} \pi^{2}}}\right)\right)}{\pi^{5} T^{5}\left(1+\sqrt{1-\frac{2}{T^{2} \pi^{2}}}\right)^{5}\left(-2+\pi^{2} T^{2}\left(1+\sqrt{1-\frac{2}{T^{2} \pi^{2}}}\right)\right) .}
$$

Plugging this into (15) we obtain the free energy as function of the temperature

$$
\begin{aligned}
F & =-\frac{\operatorname{Vol}\left(S^{3}\right) \operatorname{Vol}\left(S^{5}\right)}{16 \pi G_{10}}\left[\frac{1}{8} \pi^{2} T^{2}\left(1+\sqrt{1-\frac{2}{\pi^{2} T^{2}}}\right)^{2}\left(-3+\pi^{2} T^{2}\left(1+\sqrt{1-\frac{2}{\pi^{2} T^{2}}}\right)\right)\right. \\
& \left.+\gamma \frac{15}{4}\left(1+2 \pi^{2} T^{2}\left(-14+3 \sqrt{1-\frac{2}{T^{2} \pi^{2}}}\right)+\pi^{4} T^{4}\left(34-30 \sqrt{1-\frac{2}{T^{2} \pi^{2}}}\right)\right)\right] .
\end{aligned}
$$


The entropy is now given by $S=-\frac{\partial F}{\partial T}$ and coincides exactly with the expression given in [12]. It is very interesting that a careful calculation taking into account also the corrections to the metric gives the same results as just plugging in the uncorrected metric into the $R^{4}$ term. Noting that the corrections are rather more complicated that in the flat case considered in [7] and [9] this seems even more a miracle. So the features of the geometry at $O\left(\alpha^{\prime 3}\right)$ are not relevant to its thermodynamics at this order. It would be interesting to have a better physical understanding of this property. Another interesting point would be to compare these expressions with a two loop calculation in field theory.

\section{Acknowledgements}

I would like to thank H. Balasin and E. Lopez for discussions. 


\section{References}

[1] J. Maldacena, Adv. Theor. Math. Phys. 2 (1998) 231.

[2] S. S. Gubser, I. R. Klebanov and A. M. Polyakov, Phys.Lett. B428 (1998) 105; E. Witten, Adv.Theor.Math.Phys. 2 (1998) 253.

[3] E. Witten, Adv.Theor.Math.Phys. 2 (1998) 505.

[4] G. Horowitz and S. Ross,J.High Energy Phys. 9804 (1998) 015.

[5] S. S. Gubser, I. R. Klebanov and A. W. Peet, Phys.Rev. D54 (1996) 3915.

[6] I. R. Klebanov, "From Threebranes to Large N Gauge Theories", hep-th/9901018.

[7] S. S. Gubser, I. R. Klebanov and A. A. Tseytlin, Nucl.Phys. B534 (1998) 202.

[8] T. Banks and M. B. Green, J.High Energy Phys. 9805 (1998) 002.

[9] J. Pawelczyk and S. Theisen, J.High Energy Phys. 9809 (1998) 010.

[10] A. Fotopoulos and T. R. Taylor, "Comment on two loop free energy in N=4 supersymmetric Yang-Mills theory at finite temperature", hep-th/9811224.

[11] S. W. Hawking and D. N. Page, Commun. Math. Phys. 87 (1983) 577.

[12] Y. Gao and M. Li, "Large N Strong/Weak Coupling Phase Transition and the Correspondence Principle", hep-th/9810053. 